Appl. Set-Valued Anal. Optim. 4 (2022), No. 1, pp. 83-93

Available online at http://asvao.biemdas.com

https://doi.org/10.23952/asvao.4.2022.1.06

\title{
GENERALIZED REGULARIZED GAP FUNCTIONS AND ERROR BOUNDS FOR GENERALIZED VECTOR VARIATIONAL-LIKE INEQUALITIES
}

\author{
MOHD ASIF $^{1}$, ZUBAIR KHAN $^{2}$, FEEROZ BABU $^{2, *}$, ABDUL FAIZ ANSARI $^{3}$ \\ ${ }^{1}$ Department of Mathematics and Statistics, Integral University, Lucknow 226 026, India \\ ${ }^{2}$ Department of Applied Mathematics, Aligarh Muslim University, Aligarh, 202 002, India \\ ${ }^{3}$ Department of Mathematics and Astronomy, University of Lucknow, Lucknow 226007 , India
}

\begin{abstract}
This paper aims to study the generalized vector variational-like inequalities (in short, GVVLI). A gap function and a generalized regularized gap function which are independent of the scalarization parameter for the GVVLI. A result on error bounds for the GVVLI with generalized regularized gap functions without any Lipschitz continuity is presented. Finally, we give examples to illustrate our results. The results presented in the paper improve and generalize some known results in the literature.
\end{abstract}

Keywords. Error bounds; Gap functions; Monotone operators; Vector variational inequalities.

\section{INTRODUCTION}

A vector variational inequality (in short, VVI) was initially originated by Giannessi [1], who developed the concept of VVI in the setting of finite-dimensional spaces. VVI plays a fruitful role in operation research, economics, optimization, etc. Since the last three decades, many generalizations of VVIs have been considered and formulated to solve different types of problems, such as equilibrium problems, boundary value problems, vector optimization problems; see, e.g., $[2,3,4,5,6]$ and the references therein.

In the study of variational inequalities, gap functions provide more advantages than other methods. Gap functions play an important role to reduce variational inequalities into optimization problems, which are easy to handle from viewpoint of computation. There are several known methods to construct gap functions for VVIs in the literature; see, for example, $[7,8,9,10,11]$ and the references therein. One of the well-formed methods to establish a gap function for VVI is the scalarization method. Generated gap functions from this method depends on some scalarization parameters. In 2014, Sun and Chai [12] proposed the scalarvalued gap function for generalized vector variational inequalities in the absence of scalarization parameters. On the other hand, Fukushima [13] introduced a regularized gap function for classical variational inequalities. Further, Wu et al. [14] extend the Fukushima's work by introducing a generalized regularized gap function for classical variational inequalities. Motivated by $[13,14]$, we in this paper introduce a new type of generalized regularized gap functions

\footnotetext{
${ }^{*}$ Corresponding author.

E-mail addresses: asifzakir007@gmail.com (M. Asif), zubkhan403@gmail.com (Z. Khan), firoz77b@gmail. com (F. Babu), imfaizofficial@gmail.com (A.F. Ansari).

Received October 2, 2021; Accepted December 5, 2021.
} 
for generalized vector variational-like inequalities (in short, GVVLI) without any scalarization parameter and any Lipschitz continuity of monotone operator.

Gap functions provide efficient methods to establish error bounds for variational inequalities, and error bounds play a key role in investigating the convergence analysis of optimization problems and variational inequalities. Error bounds give an upper estimate of the distance between the feasible point and solution set of the considered problem. For more details on error bounds, we refer to $[15,16,17,18,19,20]$ and the references therein.

We organized this paper as follows. In Section 2, we gather all the necessary definitions related to monotonicity and convexity. Section 3 is devoted to the formulation of the generalized vector variational-like inequalities and generalized variational-like inequalities with their special cases. In Section 4, without any scalarization techniques and any Lipschitz continuity, we propose a gap function and its generalized regularized version for the GVVLI with some examples. In Section 5, we use the generalized regularized gap function to establish error bounds for the GVVLI. In the last section, Section 6, we provide an example that helps to understand the numerical applicability of our proposed results.

\section{PRELIMINARIES}

Let $K$ be a nonempty subset of $\mathbb{R}^{n}$. Let $\langle\cdot, \cdot\rangle: \mathbb{R}^{n} \times \mathbb{R}^{n} \rightarrow \mathbb{R}$ be an inner product, and let

$\|$.$\| be the corresponding norm. Consider the vector-valued mappings A_{j}, B_{j}: \mathbb{R}^{n} \rightarrow \mathbb{R}^{n}(j=$ $1,2, \ldots, m)$ and a real valued mapping $h_{j}: \mathbb{R}^{n} \rightarrow \mathbb{R}(j=1,2, \ldots, m)$. For abbreviation, let

$$
A:=\left(A_{1}, A_{2}, \ldots, A_{m}\right), B:=\left(B_{1}, B_{2}, \ldots, B_{m}\right) \text { and } H:=\left(h_{1}, h_{2}, \ldots, h_{m}\right),
$$

and for every $p, q \in \mathbb{R}^{n}$, we denote

$$
\langle A(p), q\rangle:=\left(\left\langle A_{1}(p), q\right\rangle,\left\langle A_{2}(p), q\right\rangle, \ldots,\left\langle A_{m}(p), q\right\rangle\right) .
$$

Definition 2.1. A mapping $\eta: K \times K \rightarrow \mathbb{R}^{n}$ is said to be skew if, for all $p, q \in K$,

$$
\eta(p, q)+\eta(q, p)=0 .
$$

Motivated by Verma [21], we define the pseudocontractivity in terms of the mapping $\eta$ : $K \times K \rightarrow \mathbb{R}^{n}$.

Definition 2.2. A vector-valued mapping $F: \mathbb{R}^{n} \rightarrow \mathbb{R}^{n}$ is said to be

(a) [21] $(\rho, \eta)$-strongly monotone if, for all $p, q \in \mathbb{R}^{n}$, there exists a positive integer $\rho$ such that

$$
\langle F(p)-F(q), \eta(p, q)\rangle \geq \rho\|p-q\|^{2} .
$$

(b) $(\varsigma, \eta)$-strongly pseudocontractive if, for all $p, q \in \mathbb{R}^{n}$, there exists a positive integer $\varsigma$ such that

$$
\langle F(p)-F(q), \eta(p, q)\rangle \leq \varsigma\|p-q\|^{2} .
$$

Definition 2.3. [22] A real-valued mapping $f: \mathbb{R}^{n} \rightarrow \mathbb{R}$ is said to be convex if, for all $p, q \in \mathbb{R}^{n}$ and all $0 \leq \lambda \leq 1$,

$$
f(\lambda p+(1-\lambda) q) \leq \lambda f(p)+(1-\lambda) f(q) .
$$

If $-f$ is convex, then $f$ is said to be concave. Moreover, if $f$ is both convex and concave, then $f$ is said to be affine.

The following definition of the convexity can be adapted to vector-valued mappings. 
Definition 2.4. A vector-valued mapping $F: \mathbb{R}^{n} \rightarrow \mathbb{R}^{m}$ defined by $F=\left(f_{1}, f_{2}, \ldots, f_{m}\right)$ is said to be convex if, for each $j=1,2, \ldots, m$, mapping $f_{j}: \mathbb{R}^{n} \rightarrow \mathbb{R}$ is convex on $\mathbb{R}^{n}$.

\section{Generalized Vector VARiational-Like Inequalities}

Let $K$ be a nonempty, closed, and convex subset of $\mathbb{R}^{n}$. Let $A_{j}, B_{j}: K \rightarrow \mathbb{R}^{n}, \eta: K \times K \rightarrow \mathbb{R}^{n}$ be vector-valued mappings, and let $h_{j}: K \rightarrow \mathbb{R}(j=1,2, \ldots, m)$ be real valued mappings.

In this section, we propose the following generalized vector variational-like inequality, which is to

$$
(G V V L I)\left\{\begin{array}{l}
\text { find } p^{*} \in K \text { such that } \\
\left\langle A\left(p^{*}\right)-B\left(p^{*}\right), \eta\left(p, p^{*}\right)\right\rangle+H(p)-H\left(p^{*}\right) \notin-\text { int } \mathbb{R}_{+}^{m}, \forall p \in K,
\end{array}\right.
$$

where $\mathbb{R}_{+}^{m}$ is the nonnegative orthant of $\mathbb{R}^{m}$, and int $\mathbb{R}_{+}^{m}$ is an interior of $\mathbb{R}_{+}^{m}$. We denote by $\operatorname{sol}(G V V L I)$ the solution set of the GVVLI. If $\eta(x, y)=x-y$ for all $x, y \in K$ and $A=B$, then the GVVLI reduces to the generalized vector variational inequality, which was considered and studied by Sun and Chai [12]. Moreover, if $\eta(x, y)=x-y$ for all $x, y \in K, A=B$, and $h_{j}=0$ for each $j=1,2, \ldots, m$, then the GVVLI reduces to the vector variational inequality, which was proposed and studied by Giannessi [1].

For $m=1$, the GVVLI turns to the following generalized variational-like inequality (GVLI):

$$
(G V L I)\left\{\begin{array}{l}
\text { Find } p^{*} \in K \text { such that } \\
\left\langle A_{1}\left(p^{*}\right)-B_{1}\left(p^{*}\right), \eta\left(p, p^{*}\right)\right\rangle+h_{1}(p)-h_{1}\left(p^{*}\right) \geq 0, \forall p \in K .
\end{array}\right.
$$

For $j=1,2, \ldots, m,(G V L I)^{j}$ denotes the GVLI with $A_{j}$ and $h_{j}$, and $\operatorname{sol}(G V L I)^{j}$ denotes the set of solutions of $(G V L I)^{j}$.

If $H=B=0$, the origin of the nonnegative orthant, then the GVVLI reduces to the generalized vector variational-like inequality, considered and discussed by Yang and Yang [23].

Finally, we end this section by the following definition.

Definition 3.1. Let $K$ be a nonempty subset of $\mathbb{R}^{n}$. A real-valued mapping $\mathscr{G}: K \rightarrow \mathbb{R}$ is said to be a gap function for (GVVLI) if it satisfies the following assertions:

(a) $\mathscr{G}(p) \geq 0$ for every $p \in K$,

(b) $\mathscr{G}\left(p^{*}\right)=0 \Leftrightarrow p^{*} \in K \cap \operatorname{sol}(G V V L I)$.

\section{GAP FUnCTIONS FOR GVVLI}

In this section, we investigate a gap function and its generalized regularized version for the GVVLI.

4.1. Gap function. We propose the following gap function $\mathscr{G}: K \rightarrow \mathbb{R}$, which is independent of the scalarization parameter for GVVLI:

$$
\mathscr{G}(p)=\sup _{q \in K} \min _{1 \leq j \leq m}\left\{\left\langle A_{j}(p)-B_{j}(p), \eta(p, q)\right\rangle+h_{j}(p)-h_{j}(q)\right\} .
$$

Proposition 4.1. Let $K$ be a nonempty, closed, and convex subset of $\mathbb{R}^{n}$, and let $\eta: K \times K \rightarrow \mathbb{R}^{n}$ be a skew mapping. Let $\eta(p, \cdot)$ be affine for every $p \in K$, and let $h_{j}: \mathbb{R}^{n} \rightarrow \mathbb{R}$ be convex for each $j=1,2, \ldots, m$. Then the function $\mathscr{G}$ given by (4.1) is well-defined. 
Proof. Assume that $\xi(p, q)=\min _{1 \leq j \leq m}\left\{\left\langle A_{j}(p)-B_{j}(p), \eta(p, q)\right\rangle+h_{j}(p)-h_{j}(q)\right\}$. Then, $\mathscr{G}(p)=$ $\sup _{q \in K}\{\xi(p, q)\}, \forall p \in K$. Since $\eta(p, \cdot)$ is affine for every $p \in K$, and $h_{j}$ is convex for each $j=1,2, \ldots, m$, then, for every $p, q_{1}, q_{2} \in K$ and $0 \leq \alpha \leq 1$,

$$
\begin{aligned}
& \xi\left(p, \alpha q_{1}+(1-\alpha) q_{2}\right) \\
& =\min _{1 \leq j \leq m}\left\{\left\langle A_{j}(p)-B_{j}(p), \eta\left(p, \alpha q_{1}+(1-\alpha) q_{2}\right)\right\rangle+h_{j}(p)-h_{j}\left(\alpha q_{1}+(1-\alpha) q_{2}\right)\right\} \\
& \geq \min _{1 \leq j \leq m}\left\{\alpha\left\langle A_{j}(p)-B_{j}(p), \eta\left(p, q_{1}\right)\right\rangle+(1-\alpha)\left\langle A_{j}(p)-B_{j}(p), \eta\left(p, q_{2}\right)\right\rangle+\alpha h_{j}(p)\right. \\
& \left.\quad+(1-\alpha) h_{j}(p)-\alpha h_{j}\left(q_{1}\right)-(1-\alpha) h_{j}\left(q_{2}\right)\right\} \\
& =\alpha \xi\left(p, q_{1}\right)+(1-\alpha) \xi\left(p, q_{2}\right),
\end{aligned}
$$

that is, $\xi(p, \cdot)$ is concave on closed and convex subset $K$ for every $p \in K$. Then $\xi(p, \cdot)$ attains the maximum at a unique point in $K$. Hence, $\mathscr{G}$ is well defined.

Theorem 4.1. Let $K$ be a nonempty closed convex subset of $\mathbb{R}^{n}$, and let $\eta: K \times K \rightarrow \mathbb{R}^{n}$ be a skew mapping. Let $\eta(p, \cdot)$ be affine for every $p \in K$, and let $h_{j}: \mathbb{R}^{n} \rightarrow \mathbb{R}$ be convex for each $j=1,2, \ldots, m$. Then the function $\mathscr{G}: K \rightarrow \mathbb{R}$ defined by (4.1) is a gap function for $G V V L I$.

Proof. (i) Since $\mathscr{G}(p) \geq \min _{1 \leq j \leq m}\left\{\left\langle A_{j}(p)-B_{j}(p), \eta(p, q)\right\rangle+h_{j}(p)-h_{j}(q)\right\}, \forall p \in K$. By setting $q=p$, it is easy to see that $\mathscr{G}(p) \geq 0$ for all $p \in K$.

(ii) Let $p^{*} \in K$ and $\mathscr{G}\left(p^{*}\right)=0$. It follows that

$$
\sup _{q \in K} \min _{1 \leq j \leq m}\left\{\left\langle A_{j}\left(p^{*}\right)-B_{j}\left(p^{*}\right), \eta\left(p^{*}, q\right)\right\rangle+h_{j}\left(p^{*}\right)-h_{j}(q)\right\}=0 .
$$

This guarantees that

$$
\min _{1 \leq j \leq m}\left\{\left\langle A_{j}\left(p^{*}\right)-B_{j}\left(p^{*}\right), \eta\left(p^{*}, q\right)\right\rangle+h_{j}\left(p^{*}\right)-h_{j}(q)\right\} \leq 0, \forall q \in K .
$$

Then there exists a $1 \leq j_{0} \leq m$ such that the inequality above reduces to

$$
\left\langle A_{j_{0}}\left(p^{*}\right)-B_{j_{0}}\left(p^{*}\right), \eta\left(p^{*}, q\right)\right\rangle+h_{j_{0}}\left(p^{*}\right)-h_{j_{0}}(q) \leq 0, \forall q \in K .
$$

Since $\eta$ is skew, then $\left\langle A_{j_{0}}\left(p^{*}\right)-B_{j_{0}}\left(p^{*}\right), \eta\left(q, p^{*}\right)\right\rangle+h_{j_{0}}(q)-h_{j_{0}}\left(p^{*}\right) \geq 0, \forall q \in K$. It follows that

$$
\left\langle A\left(p^{*}\right)-B\left(p^{*}\right), \eta\left(q, p^{*}\right)\right\rangle+H(q)-H\left(p^{*}\right) \notin-i n t \mathbb{R}_{+}^{m}, \forall q \in K .
$$

This implies that $p^{*} \in \operatorname{sol}(G V V L I)$.

Conversely, let $p^{*}$ be a solution of GVVLI. Then, there exists a $1 \leq j_{0} \leq m$ such that

$$
\left\langle A_{j_{0}}\left(p^{*}\right)-B_{j_{0}}\left(p^{*}\right), \eta\left(q, p^{*}\right)\right\rangle+h_{j_{0}}(q)-h_{j_{0}}\left(p^{*}\right) \geq 0, \forall q \in K
$$

Since $\eta$ is a skew mapping, one concludes that

$$
\left\langle A_{j_{0}}\left(p^{*}\right)-B_{j_{0}}\left(p^{*}\right), \eta\left(p^{*}, q\right)\right\rangle+h_{j_{0}}\left(p^{*}\right)-h_{j_{0}}(q) \leq 0, \forall q \in K
$$

and

$$
\min _{1 \leq j \leq m}\left\{\left\langle A_{j}\left(p^{*}\right)-B_{j}\left(p^{*}\right), \eta\left(p^{*}, q\right)\right\rangle+h_{j}\left(p^{*}\right)-h_{j}(q)\right\} \leq 0, \forall q \in K .
$$

It follows that $\mathscr{G}\left(p^{*}\right) \leq 0$. In view of $\mathscr{G}(p) \geq 0, \forall p \in K$, one has $\mathscr{G}\left(p^{*}\right)=0$. This completes the proof. 
Example 4.1. Consider a closed convex subset $K=[-1,1] \times[-1,1]$ of $\mathbb{R}^{2}$. For $n=1$ and $m=2$, define four mappings $A_{1}, A_{2}: K \rightarrow \mathbb{R}^{2}$ and $B_{1}, B_{2}: K \rightarrow \mathbb{R}^{2}$ by

$$
A_{1}(p)=\left(-3 p_{1}, 1-4 p_{2}\right) \quad \text { and } \quad A_{2}(p)=\left(-3 p_{1}-p_{1}^{3},-8 p_{2}\right), \quad \forall p=\left(p_{1}, p_{2}\right) \in K,
$$

and

$$
B_{1}(p)=\left(-2 p_{1}, 1-4 p_{2}\right) \quad \text { and } \quad B_{2}(p)=\left(-p_{1},-8 p_{2}\right), \quad \forall p=\left(p_{1}, p_{2}\right) \in K .
$$

Define three mappings $h_{1}, h_{2}: K \rightarrow \mathbb{R}$ and $\eta: K \times K \rightarrow \mathbb{R}^{2}$ by

$$
h_{1}(p)=p_{1}^{2} \quad \text { and } \quad h_{2}(p)=p_{1}^{4} \quad \eta(p, q)=q-p, \quad \forall p=\left(p_{1}, p_{2}\right), \quad \forall q=\left(q_{1}, q_{2}\right) \in K .
$$

Note that $h_{1}$ and $h_{2}$ are convex on $K$, and $\eta$ is skew and affine on the second argument. Then GVLIs are given by

$$
\begin{aligned}
\operatorname{sol}(\mathrm{GVLI})^{1} & =\left\{p \in K:\left\langle A_{1}(p)-B_{1}(p), \eta(q, p)\right\rangle+h_{1}(q)-h_{1}(p) \geq 0, \forall q \in K\right\} \\
& =\left\{p \in K:-p_{1}\left(p_{1}-q_{1}\right)+q_{1}^{2}-p_{1}^{2} \geq 0, \forall q \in K\right\} \\
& =\{(0,0)\},
\end{aligned}
$$

and

$$
\begin{aligned}
& \operatorname{sol}(\mathrm{GVLI})^{2}=\left\{p \in K:\left\langle A_{2}(p)-B_{2}(p), \eta(q, p)\right\rangle+h_{2}(q)-h_{2}(p) \geq 0, \forall q \in K\right\} \\
& =\left\{p \in K:-\left(2 p_{1}+p_{1}^{3}\right)\left(p_{1}-q_{1}\right)+q_{1}^{4}-p_{1}^{4} \geq 0, \forall q \in K\right\} \\
& =\{(0,0)\} \text {. }
\end{aligned}
$$

Therefore, $\cap_{j=1}^{2} \operatorname{sol}(G V L I)^{j}=\{(0,0)\} \neq \emptyset$. From the definition of function $\mathscr{G}: \mathbb{R}^{2} \rightarrow \mathbb{R}$, we obtain

$$
\begin{aligned}
\mathscr{G}(p) & =\sup _{q \in[-1,1]} \min _{1 \leq j \leq m}\left\{\left\langle A_{j}(p)-B_{j}(p), \eta(p, q)\right\rangle+h_{j}(p)-h_{j}(q)\right\} \\
& =\sup _{q \in[-1,1]}\left\{p_{1}\left(p_{1}-q_{1}\right)+p_{1}^{2}-q_{1}^{2}\right\} \\
& =\frac{9}{4} p_{2}^{2} .
\end{aligned}
$$

Hence, $\mathscr{G}$ is a gap function for GVLI.

4.2. Generalized regularized gap. Let $\mathscr{F}: K \times K \rightarrow \mathbb{R}$ be a mapping. For $\alpha>0$, we define a generalized regularized gap function $\mathscr{G}_{\alpha}: K \rightarrow \mathbb{R}$ for GVVLI:

$$
\mathscr{G}_{\alpha}(p)=\sup _{q \in K}\left\{\min _{1 \leq j \leq m}\left\{\left\langle A_{j}(p)-B_{j}(p), \eta(p, q)\right\rangle+h_{j}(p)-h_{j}(q)\right\}-\alpha \mathscr{F}(p, q)\right\}, \forall p \in K .
$$

Proposition 4.2. Let $K$ be a nonempty, closed, and convex subset of $\mathbb{R}^{n}$. Let $\eta: K \times K \rightarrow \mathbb{R}^{n}$ be a skew mapping, and let $\eta(p, \cdot)$ be affine. Let $\mathscr{F}(p, \cdot)$ be convex for every $p \in K$, and let $h_{j}$ be convex for each $j=1,2, \ldots, m$. Then, for $\alpha>0$, the $\mathscr{G}_{\alpha}$ given by (4.2) is well defined.

Proof. Let $\xi(p, q)=\min _{1 \leq j \leq m}\left\{\left\langle A_{j}(p)-B_{j}(p), \eta(p, q)\right\rangle+h_{j}(p)-h_{j}(q)\right\}$. Then

$$
\mathscr{G}_{\alpha}(p)=\sup _{q \in K}\{\xi(p, q)-\alpha \mathscr{F}(p, q)\}, \quad \forall p \in K .
$$

Since $\eta(p, \cdot)$ is affine for every $p \in K$, and $h_{j}$ is convex for each $j=1,2, \ldots, m$, then it follows from Proposition 4.1 that $\xi(p, \cdot)$ is concave. From the convexity of $\mathscr{F}(p, \cdot), \xi(p, \cdot)-\alpha \mathscr{F}(p, \cdot)$ 
is concave for every $p \in K$. Since $K$ is closed and convex, then function $\xi(p, \cdot)-\alpha \mathscr{F}(p, \cdot)$ attains its maximum at the unique point in $K$. Thus $\mathscr{G}_{\alpha}$ is well defined.

From now on, we present some assumptions for the mapping $\mathscr{F}: K \times K \rightarrow \mathbb{R}$ as follows.

(P1) $\mathscr{F}$ is continuously differentiable on $K \times K$;

(P2) $\mathscr{F}$ is non-negative on $K \times K$;

(P3) $\mathscr{F}(\mathfrak{r}, \cdot)$ is strongly convex on subset $K$ for every $\mathfrak{r} \in K$, i.e., $\exists \ell>0$ such that

$$
\mathscr{F}(\mathfrak{r}, p)-\mathscr{F}(\mathfrak{r}, q) \geq\left\langle\nabla_{2} \mathscr{F}(\mathfrak{r}, q), p-q\right\rangle+\ell\|p-q\|^{2}, \forall p, q, \mathfrak{r} \in K ;
$$

where $\nabla_{2} \mathscr{F}$ denotes the gradient of $\mathscr{F}(p, \cdot)$ for every $p \in K$;

(P4) $\mathscr{F}(p, q)=0 \Leftrightarrow p=q$;

(P5) $\nabla_{2} \mathscr{F}(\mathfrak{r},$.$) is Lipschitz continuous for every \mathfrak{r} \in K$, i.e., $\exists \kappa>0$ such that

$$
\left\|\nabla_{2} \mathscr{F}(\mathfrak{r}, p)-\nabla_{2} \mathscr{F}(\mathfrak{r}, q)\right\| \leq \kappa\|p-q\|, \forall p, q, \mathfrak{r} \in K .
$$

Lemma 4.1. If $\mathscr{F}: K \times K \rightarrow \mathbb{R}$ satisfies (P1) - (P5), then

$$
\mathscr{F}(p, q) \leq(\kappa-\ell)\|p-q\|^{2}, \quad \forall p, q \in K .
$$

Proof. It follows from (P3) that

$$
\mathscr{F}(p, p)-\mathscr{F}(p, q) \geq\left\langle\nabla_{2} \mathscr{F}(p, q), p-q\right\rangle+\ell\|p-q\|^{2}, \forall p, q \in K,
$$

that is, $-\mathscr{F}(p, q) \geq\left\langle\nabla_{2} \mathscr{F}(p, q), p-q\right\rangle+\ell\|p-q\|^{2}, \forall p, q \in K$. This implies

$$
\mathscr{F}(p, q) \leq\left\|\nabla_{2} \mathscr{F}(p, q)\right\|\|p-q\|-\ell\|p-q\|^{2}, \forall p, q \in K .
$$

From (P5), we have $\left\|\nabla_{2} \mathscr{F}(p, p)-\nabla_{2} \mathscr{F}(p, q)\right\| \leq \kappa\|p-q\|$. In view of (P4), we obtain

$$
\left\|\nabla_{2} \mathscr{F}(p, q)\right\| \leq \kappa\|p-q\| .
$$

Using inequalities (4.4) and (4.5), we arrive at $\mathscr{F}(p, q) \leq(\kappa-\ell)\|p-q\|^{2}, \forall p, q \in K$. The proof is complete.

Theorem 4.2. Let $K$ be a nonempty, closed and convex subset of $\mathbb{R}^{n}$, and let $\eta: K \times K \rightarrow \mathbb{R}^{n}$ be a skew mapping. Let $\eta(p, \cdot)$ be affine for every $p \in K$, and let $h_{j}: \mathbb{R}^{n} \rightarrow \mathbb{R}$ be convex for each $j=1,2, \ldots, m$. If $\mathscr{F}: K \times K \rightarrow \mathbb{R}$ satisfy $(P 1)-(P 5)$, then the function $\mathscr{G}_{\alpha}(\alpha>0)$ defined by (4.2) is a gap function for GVVLI.

Proof. (i) By setting $q=p$ in (4.2), we find that, for all $p \in K$,

$$
\mathscr{G}_{\alpha}(p)=\sup _{q \in K}\left\{\min _{1 \leq j \leq m}\left\{\left\langle A_{j}(p)-B_{j}(p), \eta(p, q)\right\rangle+h_{j}(p)-h_{j}(q)\right\}-\alpha \mathscr{F}(p, q)\right\} \geq 0 .
$$

(ii) Let $\xi(p, q)=\min _{1 \leq j \leq m}\left\{\left\langle A_{j}(p)-B_{j}(p), \eta(p, q)\right\rangle+h_{j}(p)-h_{j}(q)\right\}, p^{*} \in K$ and $\mathscr{G}_{\alpha}\left(p^{*}\right)=0$. Then, $\xi\left(p^{*}, q\right) \leq \alpha \mathscr{F}\left(p^{*}, q\right)$ for all $q \in K$. For random $p \in K$ and every $0<\lambda<1$, let $q=$ $p+\lambda\left(p^{*}-p\right)$ for all $p \in K$. It follows from Lemma 4.1 that

$$
\begin{aligned}
\xi\left(p^{*}, p+\lambda\left(p^{*}-p\right)\right) & \leq \alpha \mathscr{F}\left(p^{*}, p+\lambda\left(p^{*}-p\right)\right) \\
& \leq \alpha(\kappa-\ell)\left\|p+\lambda\left(p^{*}-p\right)-p^{*}\right\|^{2} \\
& =\alpha(\kappa-\ell)(1-\lambda)^{2}\left\|p-p^{*}\right\|^{2}
\end{aligned}
$$


that is,

$$
\begin{aligned}
& \min _{1 \leq j \leq m}\left\{\left\langle A_{j}\left(p^{*}\right)-B_{j}\left(p^{*}\right), \eta\left(p^{*}, p+\lambda\left(p^{*}-p\right)\right)\right\rangle+h_{j}\left(p^{*}\right)-h_{j}\left(p+\lambda\left(p^{*}-p\right)\right)\right\} \\
& \leq \alpha(\kappa-\ell)(1-\lambda)^{2}\left\|p-p^{*}\right\|^{2} .
\end{aligned}
$$

Since, for each $j=1,2, \ldots, m, h_{j}$ is convex, and for every $p \in K, \eta(p, \cdot)$ is affine, then

$$
\begin{aligned}
& \min _{1 \leq j \leq m}\left\{\left\langle A_{j}\left(p^{*}\right)-B_{j}\left(p^{*}\right), \lambda \eta\left(p^{*}, p^{*}\right)+(1-\lambda) \eta\left(p^{*}, p\right)\right\rangle+h_{j}\left(p^{*}\right)\right. \\
& \left.\quad-\lambda h_{j}\left(p^{*}\right)-(1-\lambda) h_{j}(p)\right\} \\
& \leq \alpha(\kappa-\ell)(1-\lambda)^{2}\left\|p-p^{*}\right\|^{2}
\end{aligned}
$$

Hence,

$$
\begin{aligned}
& (1-\lambda) \min _{1 \leq j \leq m}\left\{\left\langle A_{j}\left(p^{*}\right)-B_{j}\left(p^{*}\right), \eta\left(p^{*}, p\right)\right\rangle+h_{j}\left(p^{*}\right)-h_{j}(p)\right\} \\
& \leq \alpha(\kappa-\ell)(1-\lambda)^{2}\left\|p-p^{*}\right\|^{2}
\end{aligned}
$$

and

$$
\begin{aligned}
& \min _{1 \leq j \leq m}\left\{\left\langle A_{j}\left(p^{*}\right)-B_{j}\left(p^{*}\right), \eta\left(p^{*}, p\right)\right\rangle+h_{j}\left(p^{*}\right)-h_{j}(p)\right\} \\
& \leq \alpha(\kappa-\ell)(1-\lambda)\left\|p-p^{*}\right\|^{2} .
\end{aligned}
$$

By taking $\lambda \rightarrow 1$, we arrive at $\min _{1 \leq j \leq m}\left\{\left\langle A_{j}\left(p^{*}\right)-B_{j}\left(p^{*}\right), \eta\left(p^{*}, p\right)\right\rangle+h_{j}\left(p^{*}\right)-h_{j}(p)\right\} \leq 0$. Thus, there exists a $1 \leq j_{0} \leq m$ such that

$$
\left\langle A_{j_{0}}\left(p^{*}\right)-B_{j_{0}}\left(p^{*}\right), \eta\left(p^{*}, p\right)\right\rangle+h_{j_{0}}\left(p^{*}\right)-h_{j_{0}}(p) \leq 0 .
$$

It follows that $\left\langle A\left(p^{*}\right)-B\left(p^{*}\right), \eta\left(p, p^{*}\right)\right\rangle+H(p)-H\left(p^{*}\right) \notin$ int $\mathbb{R}_{+}^{m}, \forall p \in K$. Hence, we obtain $p^{*} \in \operatorname{sol}(G V V L I)$.

Conversely, we assume that $p^{*}$ is a solution of GVVLI. Then, there exists a $1 \leq i_{0} \leq m$ such that

$$
\left\langle A_{j_{0}}\left(p^{*}\right)-B_{j_{0}}\left(p^{*}\right), \eta\left(q, p^{*}\right)\right\rangle+h_{j_{0}}(q)-h_{j_{0}}\left(p^{*}\right) \geq 0, \forall q \in K
$$

From the skew property of $\eta$, we have

$$
\left\langle A_{j_{0}}\left(p^{*}\right)-B_{j_{0}}\left(p^{*}\right), \eta\left(p^{*}, q\right)\right\rangle+h_{j_{0}}\left(p^{*}\right)-h_{j_{0}}(q) \leq 0, \forall q \in K
$$

and hence,

$$
\min _{1 \leq j \leq m}\left\{\left\langle A_{j}\left(p^{*}\right)-B_{j}\left(p^{*}\right), \eta\left(p^{*}, q\right)\right\rangle+h_{j}\left(p^{*}\right)-h_{j}(q)\right\} \leq 0, \forall q \in K .
$$

that is, $\xi\left(p^{*}, q\right) \leq 0$. This together with (4.3) yields that $\mathscr{G}_{\alpha}\left(p^{*}\right) \leq 0$. But $\mathscr{G}_{\alpha}\left(p^{*}\right) \geq 0$ for all $p \in K$. So, $\mathscr{G}_{\alpha}\left(p^{*}\right)=0$. The proof is complete.

Example 4.2. Consider $K, A_{1}, A_{2}, B_{1}, B_{2}, h_{1}, h_{2}$, and $\eta$ as same as in Example 4.1 and define $\mathscr{F}: \mathbb{R}^{2} \times \mathbb{R}^{2} \rightarrow \mathbb{R}$ by

$$
\mathscr{F}(p, q)=\frac{1}{2}\left(p_{1}-q_{1}\right)^{2}+\frac{1}{2}\left(p_{2}-q_{2}\right)^{2}, \quad \forall p=\left(p_{1}, p_{2}\right), \forall q=\left(q_{1}, q_{2}\right) \in K .
$$


Clearly, $\mathscr{F}$ satisfies conditions (P1) - (P5). From the definition of the function $\mathscr{G}_{\alpha}: \mathbb{R}^{2} \rightarrow \mathbb{R}$ for $\alpha>0$, we have

$$
\begin{aligned}
\mathscr{G}_{\alpha}(p) & =\sup _{q \in[-1,1]}\left\{\min _{1 \leq j \leq m}\left\{\left\langle A_{j}(p)-B_{j}(p), \eta(p, q)\right\rangle+h_{j}(p)-h_{j}(q)\right\}-\alpha \mathscr{F}(p, q)\right\} \\
& =\sup _{q \in[-1,1]}\left\{p_{1}\left(p_{1}-q_{1}\right)+p_{1}^{2}-q_{1}^{2}-\frac{\alpha}{2}\left(p_{1}-q_{1}\right)^{2}-\frac{\alpha}{2}\left(p_{2}-q_{2}\right)^{2}\right\} \\
& =\frac{9}{2(\alpha+2)} p_{1}^{2} .
\end{aligned}
$$

Thus, $\mathscr{G}_{\alpha}$ is a gap function for GVVLI.

\section{ERROR BOUNDS}

In this section, we obtain the error bounds for GVVLI with the help of the gap function and the generalized regularized gap function given by (4.2).

Lemma 5.1. If $A_{j}: \mathbb{R}^{n} \rightarrow \mathbb{R}^{n}$ is $\left(\rho_{j}, \eta\right)$-strongly monotone, and $B_{j}: \mathbb{R}^{n} \rightarrow \mathbb{R}^{n}$ is $\left(\varsigma_{j}, \eta\right)$-strongly pseudocontractive over $K$ such that $\rho_{j}>\varsigma_{j}$ for each $j=1,2, \ldots, m$, then $A_{j}-B_{j}$ is $\left(\rho_{j}-\varsigma_{j}, \eta\right)$ strongly monotone.

Proof. By the additivity property of inner products, for every $p, q \in \mathbb{R}^{n}$, we have

$$
\begin{aligned}
& \left\langle A_{j}(p)-B_{j}(p), \eta(p, q)\right\rangle \\
& =\left\langle\left(A_{j}(p)-B_{j}(p)\right)-\left(A_{j}(q)-B_{j}(q)\right)+\left(A_{j}(q)-B_{j}(q)\right), \eta(p, q)\right\rangle \\
& =\left\langle\left(A_{j}(p)-A_{j}(q)\right)-\left(B_{j}(p)-B_{j}(q)\right), \eta(p, q)\right\rangle+\left\langle\left(A_{j}(q)-B_{j}(q)\right), \eta(p, q)\right\rangle \\
& =\left\langle\left(A_{j}(p)-A_{j}(q)\right), \eta(p, q)\right\rangle-\left\langle\left(B_{j}(p)-B_{j}(q)\right), \eta(p, q)\right\rangle+\left\langle\left(A_{j}(q)-B_{j}(q)\right), \eta(p, q)\right\rangle .
\end{aligned}
$$

From the $\left(\rho_{j}, \eta\right)$-strongly monotonicity and $\left(\varsigma_{j}, \eta\right)$-strongly pseudocontractivity of $A_{j}$ and $B_{j}$, respectively, we conclude

$$
\left\langle A_{j}(p)-B_{j}(p), \eta(p, q)\right\rangle \geq\left\langle A_{j}(q)-B_{j}(q), \eta(p, q)\right\rangle+\left(\rho_{j}-\varsigma_{j}\right)\|p-q\|^{2} .
$$

This completes the proof.

Theorem 5.1. Let $\eta: K \times K \rightarrow \mathbb{R}^{n}$ be a skew mapping, and let $\eta(p, \cdot)$ be affine for every $p \in K$. For each $j=1,2, \ldots, m$, let $A_{j}: \mathbb{R}^{n} \rightarrow \mathbb{R}^{n}$ be $\left(\rho_{j}, \eta\right)$-strongly monotone, $B_{j}: \mathbb{R}^{n} \rightarrow \mathbb{R}^{n}$ be $\left(\varsigma_{j}, \eta\right)$-strongly pseudocontractive with $\rho_{j}>\varsigma_{j}$, respectivelyover $K$, and $h_{j}: \mathbb{R}^{n} \rightarrow \mathbb{R}$ be convex. Let $\mathscr{F}: K \times K \rightarrow \mathbb{R}$ satisfy (P1)-(P5). Further, suppose that $\bigcap_{j=1}^{m} \operatorname{sol}(G V L I)^{j}$ is nonempty, $\vartheta=\min _{1 \leq j \leq m}\left(\rho_{j}-\varsigma_{j}\right)$, and $\alpha, \kappa, \ell>0$ such that $\vartheta>\alpha(\kappa-\ell)$. Then

$$
d(p, \operatorname{sol}(G V V L I)) \leq \frac{1}{\sqrt{(\vartheta-\alpha(\kappa-\ell))}} \sqrt{\mathscr{G}_{\alpha}(p)}, \quad \forall p \in K,
$$

where $d(p, \operatorname{sol}(G V V L I))$ is the distance between sol(GVVLI) and point $p$.

Proof. By (4.3) and Lemma 4.1, for every $q \in K$, we have

$$
\mathscr{G}_{\alpha}(p) \geq \xi(p, q)-\alpha \mathscr{F}(p, q) \geq \xi(p, q)-\alpha(\kappa-\ell)\|p-q\|^{2} .
$$


Since every $(G V L I)^{j}$ has same solution as $\bigcap_{j=1}^{m} \operatorname{sol}(G V L I)^{j}$, which is nonempty. If $p^{*} \in K$ is a solution, then $p^{*}$ is the solution to GVVLI. Note that $\mathscr{G}_{\alpha}(p) \geq \min _{1 \leq j \leq m}\left\{\left\langle A_{j}(p)-\right.\right.$ $\left.\left.B_{j}(p), \eta\left(p, p^{*}\right)\right\rangle+h_{j}(p)-h_{j}\left(p^{*}\right)\right\}-\alpha(\kappa-\ell)\left\|p-p^{*}\right\|^{2}$. Suppose

$$
\begin{aligned}
& \left\langle A_{j}(p)-B_{j}(p), \eta\left(p, p^{*}\right)\right\rangle+h_{j}(p)-h_{j}\left(p^{*}\right) \\
& =\min _{1 \leq j \leq m}\left\{\left\langle A_{j}(p)-B_{j}(p), \eta\left(p, p^{*}\right)\right\rangle+h_{j}(p)-h_{j}\left(p^{*}\right)\right\} .
\end{aligned}
$$

By using Lemma 5.1, we have

$$
\mathscr{G}_{\alpha}(p) \geq\left\langle A_{j}\left(p^{*}\right)-B_{j}\left(p^{*}\right), \eta\left(p, p^{*}\right)\right\rangle+h_{j}(p)-h_{j}\left(p^{*}\right)+(\vartheta-\alpha(\kappa-\ell))\left\|p-p^{*}\right\|^{2} .
$$

Since $p^{*}$ is the solution of $(G V L I)^{j}$, then

$$
\left\langle A_{j}\left(p^{*}\right)-B_{j}\left(p^{*}\right), \eta\left(p, p^{*}\right)\right\rangle+h_{j}(p)-h_{j}\left(p^{*}\right) \geq 0 .
$$

Form (5.1) and (5.2), we obtain $\mathscr{G}_{\alpha}(p) \geq(\vartheta-\alpha(\kappa-\ell))\left\|p-p^{*}\right\|^{2}$. It follows that

$$
\left\|p-p^{*}\right\| \leq \frac{1}{\sqrt{(\vartheta-\alpha(\kappa-\ell))}} \sqrt{\mathscr{G}_{\alpha}(p)}
$$

This implies that

$$
d(p, \operatorname{sol}(G V V L I)) \leq \frac{1}{\sqrt{(\vartheta-\alpha(\kappa-\ell))}} \sqrt{\mathscr{G}_{\alpha}(p)}
$$

This completes the proof.

Similarly, by using gap function $\mathscr{G}$, we obtain the following error bound for (GVVLI).

Corollary 5.1. Let $\eta: K \times K \rightarrow \mathbb{R}^{n}$ be a skew mapping, and let $\eta(p, \cdot)$ be affine for every $p \in K$. Let $A_{1}: \mathbb{R}^{n} \rightarrow \mathbb{R}^{n}$ be $\left(\rho_{1}, \eta\right)$-strongly monotone. Lt $B_{1}: \mathbb{R}^{n} \rightarrow \mathbb{R}^{n}$ be $\left(\varsigma_{1}, \eta\right)$-strongly pseudocontractive with $\rho_{1}>\varsigma_{1}$, respectively over $K$, and let $h_{1}: \mathbb{R}^{n} \rightarrow \mathbb{R}$ be convex. If $\operatorname{sol}(G V L I)^{1}$ is nonempty and $\vartheta=\rho_{1}-\varsigma_{1}$, then $d(p, \operatorname{sol}(G V L I)) \leq \sqrt{\frac{\mathscr{G}(p)}{\vartheta}}, \forall p \in K$, where $d(p, \operatorname{sol}(G V L I))$ is the distance between sol(GVLI) and point p.

\section{Numerical Result}

Example 6.1. Let $K=[-1,1] \subseteq \mathbb{R}$ be a closed and convex subset, and let $\eta: \mathbb{R} \times \mathbb{R} \rightarrow \mathbb{R}$, be a mapping given by

$$
\eta(p, q)=q-p, \quad \forall p, q \in K
$$

For $n=1, m=2$, define four mappings $A_{1}, A_{2}: K \rightarrow \mathbb{R}$ and $B_{1}, B_{2}: K \rightarrow \mathbb{R}$ by

$$
A_{1}(p)=-3 p \quad \text { and } \quad A_{2}(p)=-3 p-p^{3} \quad \forall p \in K
$$

and

$$
B_{1}(p)=-2 p \quad \text { and } \quad B_{2}(p)=-p \quad \forall p \in K
$$

Then

$$
A(p)=\left(-3 p,-3 p-p^{3}\right) \quad \text { and } \quad B(p)=(-2 p,-p), \quad \forall p \in K .
$$

Note that $A_{1}$ and $A_{2}$ are $(3, \eta)$-strongly monotone and $(3, \eta)$-strongly monotone, respectively, and $B_{1}$ and $B_{2}$ are $(2, \eta)$-strongly pseudocontractive and $(1, \eta)$-strongly pseudocontractive, respectively. Let $h_{1}, h_{2}: \mathbb{R} \rightarrow \mathbb{R}, \eta: \mathbb{R} \times \mathbb{R} \rightarrow \mathbb{R}$ and $\mathscr{F}: \mathbb{R} \times \mathbb{R} \rightarrow \mathbb{R}$ be mappings defined by

$$
h_{1}(p)=p^{2} \quad \text { and } \quad h_{2}(p)=p^{4}, \quad \forall p \in K
$$


and

$$
\mathscr{F}(p, q)=(p-q)^{2}, \quad \forall p, q \in K
$$

Notice that $h_{1}$ and $h_{2}$ are convex, $\eta$ is skew and affine on the second argument, and $\mathscr{F}$ satisfies (P1) - (P5). Clearly, $l=1, \kappa=2$, and the GVLIs are given by

$$
\begin{aligned}
\operatorname{sol}(\mathrm{GVLI})^{1} & =\left\{p \in K:\left\langle A_{1}(p)-B_{1}(p), \eta(q, p)\right\rangle+h_{1}(q)-h_{1}(p) \geq 0, \forall q \in K\right\} \\
& =\left\{p \in K:-p(p-q)+q^{2}-p^{2} \geq 0, \forall q \in K\right\}=\{0\}
\end{aligned}
$$

and

$$
\begin{aligned}
\operatorname{sol}(\mathrm{GVLI})^{2} & =\left\{p \in K:\left\langle A_{2}(p)-B_{2}(p), \eta(q, p)\right\rangle+h_{2}(q)-h_{2}(p) \geq 0, \forall q \in K\right\} \\
& =\left\{p \in K:-\left(2 p+p^{3}\right)(p-q)+q^{4}-p^{4} \geq 0, \forall q \in K\right\}=\{0\} .
\end{aligned}
$$

Therefore, we have $\cap_{j=1}^{2} \operatorname{sol}(G V L I)^{j}=\{0\} \neq \emptyset$. From the definition of the function $\mathscr{G}: \mathbb{R} \rightarrow \mathbb{R}$, we obtain

$$
\begin{aligned}
\mathscr{G}(p) & =\sup _{q \in[-1,1]} \min _{1 \leq j \leq m}\left\{\left\langle A_{j}(p)-B_{j}(p), \eta(p, q)\right\rangle+h_{j}(p)-h_{j}(q)\right\} \\
& =\sup _{q \in[-1,1]} \min _{1 \leq j \leq m}\left\{p(p-q)+p^{2}-q^{2},\left(2 p+p^{3}\right)(p-q)+p^{4}-q^{4}\right\} \\
& =\sup _{q \in[-1,1]}\left\{p(p-q)+p^{2}-q^{2}\right\} \\
& =\frac{9}{4} p^{2} .
\end{aligned}
$$

Hence, $\mathscr{G}(p)$ is a gap function for GVLI. By the definition of the function $\mathscr{G}_{\alpha}: \mathbb{R} \rightarrow \mathbb{R}$ for $0<\alpha<1$, we have

$$
\begin{aligned}
\mathscr{G}_{\alpha}(p) & =\sup _{q \in[-1,1]}\left\{\min _{1 \leq j \leq m}\left\{\left\langle A_{j}(p)-B_{j}(p), \eta(p, q)\right\rangle+h_{j}(p)-h_{j}(q)\right\}-\alpha \mathscr{F}(p, q)\right\} \\
& =\sup _{q \in[-1,1]}\left\{p(p-q)+p^{2}-q^{2}-\alpha(p-q)^{2}\right\} \\
& =\frac{9}{4(1+\alpha)} p^{2} .
\end{aligned}
$$

Thus, $\mathscr{G}_{\alpha}(p)$ is a gap function for GVVLI. Therefore, all the conditions of Theorem 5.1 are satisfied. Since $0<\alpha<1, \vartheta=1, \kappa=2$, and $l=1$. Then

$$
\begin{aligned}
\frac{1}{\sqrt{(\vartheta-\alpha(\kappa-\ell))}} \sqrt{\mathscr{G}_{\alpha}(p)} & =\frac{1}{\sqrt{(1-\alpha(2-1))}} \sqrt{\frac{9 p^{2}}{4(1+\alpha)}} \\
& =\frac{3 p}{2 \sqrt{1-\alpha^{2}}}>p .
\end{aligned}
$$

\section{Acknowledgments}

The authors thank to the anonymous referees for their important suggestions and valuable comments, which improve this paper a lot. The first author was supported by UGC (201819NFO-2018-19-OBC-UTT-71260), India, and the fourth author was supported by UGC (201819MANF-2018-19-UTT-100556), India. 


\section{REFERENCES}

[1] F. Gianessi, Theorems of the alternative, quadratic programs and complimentarity problems, In: Cottle, R.W., Gianessi F., Lions J.L. (eds.) Variational Inequalities and Complimentarity Problems, pp. 151-186. Wiley, New York, 1980.

[2] Q.H. Ansari, E. Kobis, J.C. Yao, Vector Variational Inequalities and Vector Optimization, Springer, 2018.

[3] E. Köbis, M.A. Köbis, The weighted set relation: Characterizations in the convex case, J. Nonlinear Var. Anal. 5 (2021), 721-735.

[4] D.D. Hang, T.V. Su, On optimality conditions for efficient solutions in constrained vector equilibrium problems in terms of Studniarski's derivatives, J. Nonlinear Funct. Anal. 2020 (2020), Article ID 27.

[5] L.V. Nguyen, X. Qin, Weak sharpness and finite convergence for mixed variational inequalities, J. Appl. Numer. Optim. 1 (2019), 77-90.

[6] T.V. Su, On the upper C-continuity on the solution set of general vector alpha optimization problems in infinite-dimensional spaces, Commun. Optim. Theory, 2018 (2018), Article ID 2.

[7] X.Q. Yang, J.C. Yao, Gap functions and existence of solutions to set-valued vector variational inequalities, J. Optim. Theory Appl. 115 (2002), 407-417.

[8] G.Y. Chen, C.J. Goh, X.Q. Yang, On gap functions for vector variational inequalities, In: Gianessi, F. (eds.) Vector variational inequalities and vector equilibria: mathematical theories, pp. 55-70. Kluwer Academic, Boston, 2000.

[9] L.V. Nguyen, X. Qin, Some results on strongly pseudomonotone quasi-variational inequalities, Set-Valued Var. Anal. 28 (2020), 239-257.

[10] N.J. Huang, J. Li, J.C. Yao, Gap functions and existence of solutions for a system of vector equalibrium problems, J. Optim. Theory Appl. 133 (2007), 201-212.

[11] J. Li, G. Mastroeni, Vector varitional inequalities involving set-valued mappings via scalarization with applications to error bounds for gap functions, J. Optim. Theory Appl. 145 (2010), 355-372.

[12] X.K. Sun, Y. Chai, Gap functions and error bounds for generalized vector variational inequalities, Optim. Lett. 8 (2014), 1663-1673.

[13] M. Fukushima, Equaivalent differentiable optimization problems and descent methods for asymmetric variational inequality problems. Math. Program. 53 (1992), 99-110.

[14] J.H. Wu, M. Florian, P. Marcotte, A general descent framework for the monotone variational inequality problem, Math. Program. 61 (1993), 281-300.

[15] C. Charitha, J. Dutta, Regularized gap functions and error bounds for vector variational inequality, Pacific J. Optim. 6 (2010), 497-510.

[16] F. Facchinei, J.S. Pang, Finite-Dimensional Variational Inequalities and Complimentarity Problems, Springer, Berlin, 2003.

[17] G.Y. Li, K.F. Ng, Error bounds of generalized D-gap functions for nonsmooth and nonmonotone variational inequality problems, SIAM J. Optim. 20 (2009), 667-690.

[18] D. Aussel, B. Correa, M. Marechal, Gap functions for quasivariational inequalities and generalized Nash equalibrium problems, J. Optim. Theory Appl. 151 (2011), 474-488.

[19] D. Aussel, J. Dutta, On gap functions for multivalued Stampacchia variational inequalities, J. Optim. Theory Appl. 149 (2011), 513-527.

[20] Z. Khan, A.F. Ansari, Gap function with error bound for random generalized variational-like inequality problem, J. Sci. Arts 4 (2020), 917-930.

[21] R.U. Verma, Approximation solvability of a class of nonlinear set-valued variational inclusions involving (A, $\eta$ )-monotone mappings, J. Math. Anal. Appl. 337 (2008), 969-975.

[22] R.T. Rockafellar, Convex Analysis, Princeton University Press, Princeton, 1970.

[23] X.M. Yang, X.Q. Yang, Vector variational-like inequality with pseudo invexity, Optimization 55 (2006), 157170. 